\title{
Note on the existence theorem in "Emergent Quantum Mechanics and Emergent Symmetries"
}

\author{
Hans-Thomas Elze \\ Dipartimento di Fisica, Università di Pisa \\ Largo Pontecorvo 3, I-56127 Pisa, Italia \\ E-mail: elze@df.unipi.it
}

\begin{abstract}
Recently 't Hooft demonstrated that "For any quantum system there exists at least one deterministic model that reproduces all its dynamics after prequantization". An extension is presented here which covers quantum systems that are characterized by a complete set of mutually commuting Hermitian operators (beables). We introduce the symmetry of beables: any complete set of beables is as good as any other one which is obtained through a real general linear group transformation. The quantum numbers of a specific set are related to symmetry breaking initial and boundary conditions in a deterministic model. The Hamiltonian, in particular, can be taken as the emergent beable which provides the best resolution of the evolution of the model universe.
\end{abstract}

Keywords: foundations of quantum mechanics, beables, emergent quantum states PACS: 03.65.Ta, 04.60.-m, 05.20.-y

\section{Introduction}

The existence theorem of 't Hooft concerns the Schrödinger equation for a quantum system with a $d$-dimensional Hilbert space:

$$
\frac{\mathrm{d} \psi}{\mathrm{d} t}=-i \hat{H} \psi
$$

where $\hat{H}$ denotes the Hamiltonian, a $d \times d$ matrix here.

As demonstrated in Ref. [1, the dynamics of Eq. (1) is reproduced in a deterministic system with two degrees of freedom, one periodic variable, $\varphi \in[0,2 \pi[$, and another real variable, $\omega$, 
which evolve according to the classical equations of motion:

$$
\begin{aligned}
& \frac{\mathrm{d} \varphi(t)}{\mathrm{d} t}=\omega, \\
& \frac{\mathrm{d} \omega(t)}{\mathrm{d} t}=-\kappa f(\omega) f^{\prime}(\omega), \quad f(\omega):=\operatorname{det}(\hat{H}-\omega),
\end{aligned}
$$

with a parameter $\kappa>0$.

The following argument is based on the observation that $\omega$ moves exponentially fast towards one of the eigenvalues of $\hat{H}$, since multiplying $f$ by minus one times its derivative $f^{\prime}$ makes all corresponding zeros attractive 1 The initial condition for Eq. (3) determines which eigenvalue $E_{i}$ is approached, resulting in a limit cycle for $\varphi$ with period $T_{i} \equiv 2 \pi \omega_{i}^{-1}=2 \pi E_{i}^{-1}$.

In order to proceed, two auxiliary operators are introduced:

$$
\hat{p}_{\varphi}:=-i \frac{\partial}{\partial \varphi}, \quad \hat{p}_{\omega}:=-i \frac{\partial}{\partial \omega},
$$

which do not correspond to classically observable quantities. We also define the operator $\hat{h}$ :

$$
\hat{h}:=\omega \hat{p}_{\varphi}-\frac{\kappa}{2}\left\{f(\omega) f^{\prime}(\omega), \hat{p}_{\omega}\right\}
$$

with $\{x, y\}:=x y+y x$. This operator generates the evolution described by the classical equations of motion (2)-(3). Indeed, they can be written as:

$$
\begin{aligned}
& \frac{\mathrm{d} \varphi(t)}{\mathrm{d} t}=-i[\varphi(t), \hat{h}], \\
& \frac{\mathrm{d} \omega(t)}{\mathrm{d} t}=-i[\omega(t), \hat{h}],
\end{aligned}
$$

with $[x, y]:=x y-y x$. Thus, the operator formalism, which is familiar in quantum theory, turns out to be useful in this classical context as well. Remarkably, the generator $\hat{h}$ is Hermitian, despite the dissipative character of the equations motion.

It is natural to consider the Hilbert space on which these operators act and call its elements prequantum states. They can be employed as usual, if one wishes to calculate the observable properties of the classical system, which are functions $\mathcal{O}(\varphi, \omega)$.

Let us consider the evolution of those prequantum states $\psi$ which describe the trajectory of the classical system for an arbitrary but fixed initial condition:

$$
\begin{aligned}
& \psi(\varphi, \omega ; t)=\sum_{n} e^{i n \varphi} \psi_{n}(\omega ; t) \\
& \stackrel{t \rightarrow \infty}{\longrightarrow} \sum_{n} e^{i n\left(\varphi-\omega_{i} t\right)} \psi_{n}\left(\omega_{i} ; 0\right),
\end{aligned}
$$

\footnotetext{
${ }^{1}$ For an illustration of the behaviour of the right-hand side of Eq. (3), see Figure 1 of Ref. [1].
} 
where $\omega_{i}$ is the particular fixed point to which $\omega(t)$ is attracted, depending on its initial condition; the Fourier transformation takes periodicity in the angular variable into account.

Finally, we see that in a superselection sector, where the absolutely conserved "quantum number" $n$ is fixed to a particular value $n^{\prime}$, the prequantum states are directly related to the energy eigenstates of the quantum system described by Eq. (1):

$$
e^{-i E_{i} t^{\prime}} \psi\left(E_{i}\right)=e^{-i n^{\prime} \omega_{i} t} \psi_{n^{\prime}}\left(\omega_{i} ; 0\right)
$$

evolving in the usual way, with $t^{\prime}:=n^{\prime} t$. Probabilistic superpositions of such prequantum states with different $\omega_{i}$ result in (mixed) quantum states showing interference.

In conclusion, characteristic features of quantum systems described by Eq. (1) emerge here from the dissipative evolution of deterministic systems beneath. - This completes our review of the existence theorem of Ref. [1].

Our extension of the existence theorem is partly motivated by the fact that the appearance of the set of eigenvalues $\left\{E_{i}\right\}$ of the Hamiltonian in $\operatorname{det}(\hat{H}-\omega)=\prod_{i}\left(E_{i}-\omega\right)$ is puzzling, in the classical context of Eqs. (2)-(3): While the existence theorem provides a deterministic dynamics that gives rise to the quantum mechanical one, it does give neither a (classical or other) interpretation of this set of numbers nor a mechanism which determines them at the pre-quantum level. - Furthermore, the states of generic quantum mechanical objects are fully specified only by a set of quantum numbers which corresponds to a complete set of mutually commuting Hermitian operators. The present note addresses these aspects.

For additional results and discussions concerning emergent quantum mechanics, we refer the reader to Refs. [2]-[12], with further references therein.

\section{The symmetry of beables}

A complete characterization of the state of a quantum mechanical object with a finite number of degrees of freedom, generally, requires a set of simultaneous eigenvalues of a number of linearly

independent and mutually commuting Hermitian operators, $\hat{A}_{n}, n=1, \ldots, N$, which are called beables [13. These operators are here assumed to act on a finite dimensional Hilbert space.

A specific set of beables, represented by $\vec{A}:=\left(\hat{A}_{1}, \ldots, \hat{A}_{N}\right)^{t}$, can be interpreted as the set of operator valued coordinates of a point in an abstract $N$-dimensional vector space. The intrinsic physical properties of the quantum mechanical object correspond to this particular point, independently of the chosen coordinates.

Objects carrying spin and isospin or spin and orbital angular momentum provide examples with finite dimensional Hilbert spaces. Various choices of beables are possible, related to various "coupling schemes" which are well known in atomic and nuclear physics. 
Clearly, the selection of sets of beables is largely conditioned by empirical input, such as the observation of dynamical symmetries, and the attempt to obtain a concise mathematical theory of the objects under study.

However, concerning foundational issues of quantum theory, it may be useful not to limit the attention to special choices of beables. Instead, we wish to treat all possible choices on an equal footing, implementing the Symmetry of Beables:

- There is no absolute meaning attached to a given set of beables, represented by $\vec{A}$, as compared to a second one, $\overrightarrow{A^{\prime}}$, which is obtained from the former by the action of a group of symmetry transformations. The transformed set describes the quantum mechanical object just as well, in principle, provided the map is compatible with the Hilbert space properties of hermiticity, pairwise commutativity, and completeness of the beables [13].

Since operators with zero eigenvalues are not excluded, some of the $\hat{A}$ 's may not have an inverse. This limits the set of real transformation functions representing the symmetry of beables to those that can be expanded into series of multinomials in $\hat{A}$ 's. While nonlinear transformations are possible, for simplicity, we restrict ourselves to the group of linear transformations:

$$
\hat{A}_{n}^{\prime}=\sum_{m=1}^{N} M_{n m} \hat{A}_{m}, \quad M \in \mathrm{GL}(N, \mathbf{R})
$$

i.e., which can be represented by regular real $N \times N$ matrices.

We note in passing that the general linear group in $N$ dimensions contains the group of permutations for sets of $N$ elements as a subgroup. The latter plays the role of the diffeomorphism group in the context of causal sets [14, 15]. Therefore, we speculate that there might be a connection between the symmetry of beables which are attributed to quantum mechanical objects, especially atomistic "events", and the correspondent of general coordinate invariance for a fundamentally discrete spacetime.

The quantum states of the object evolve according to the Schrödinger equation (1), where the Hamiltonian presently acts on the finite dimensional Hilbert space pertaining to the set of beables. Given the symmetry, cf. Eq. (11), the Hamiltonian must be among the beables or be a linear combination of them. Otherwise they would not be complete.

In the following sections, we will show how the theory of such quantum mechanical objects can be completely reconstructed in classical terms. In particular, the Schrödinger evolution and symmetry properties are shown to emerge from a deterministic prequantum model. Particular examples of emergent quantum mechanical symmetries have also been discussed in Refs. [1, 9]. 


\section{Prequantum dynamics}

The model comprises $N$ real degrees of freedom which are periodic, $\vec{\varphi}:=\left(\varphi_{1}, \ldots, \varphi_{N}\right)^{t}, \varphi_{n} \in$ $[0,2 \pi[$, and evolve according to the classical equation of motion:

$$
\frac{\mathrm{d} \vec{\varphi}(t)}{\mathrm{d} t}=\vec{\omega}
$$

involving a second set of $N$ real degrees of freedom, $\vec{\omega}:=\left(\omega_{1}, \ldots, \omega_{N}\right)^{t}$. The equation of motion of $\vec{\omega}$ will follow in Eq. (18) below.

First, however, we introduce an auxiliary real field $F$ on the space of matrices $M$ representing the symmetry group of beables, $M \in \mathrm{GL}(N, \mathbf{R})$, which obeys the wave equation:

$$
\left(\partial_{t}+\dot{\vec{\omega}} \cdot \partial_{M \cdot \vec{A}}\right)\left(\partial_{t}-\dot{\vec{\omega}} \cdot \partial_{M \cdot \vec{A}}\right) F(M, t)=0
$$

where $\dot{\vec{\omega}}:=\mathrm{d} \vec{\omega} / \mathrm{d} t$. Parametrically, the field depends on real numbers $A_{n}^{j}, n=1, \ldots, N, j=$ $1, \ldots, d$, which can be considered to define the sets of simultaneous eigenvalues of $N$ commuting Hermitian operators, collectively denoted by $\vec{A}:=\left(\hat{A}_{1}, \ldots, \hat{A}_{N}\right)^{t}$. This is also visible in the general solution of the wave equation:

$$
F(M, t)=f(\vec{\omega}(t)-M \cdot \vec{A})+g(\vec{\omega}(t)+M \cdot \vec{A})
$$

where $f$ and $g$ are two real functions (of respective combinations of Hilbert space operators) with suitable differentiability properties, but otherwise arbitrary.

An important property of beables is that related eigenvalues are invariant under unitary transformations in Hilbert space. With hindsight, therefore, we require the field $F$ to be a scalar under such transformations.

Furthermore, the arguments $\vec{\omega}(t) \pm M \cdot \vec{A}$, which appear in the general solution (14), transform covariantly with respect to the symmetry of beables, i.e., under the transformations:

$$
\begin{aligned}
\vec{A} & \longrightarrow \vec{A}^{\prime}=S \vec{A}, \\
\vec{\omega} & \longrightarrow \vec{\omega}^{\prime}=S \vec{\omega}, \\
M & \longrightarrow M^{\prime}=S M S^{-1}, \quad S \in \mathrm{GL}(N, \mathbf{R})
\end{aligned}
$$

leaving, of course, a suitably defined scalar product of vectors invariant.

We now seek an initial condition that breaks the $\operatorname{GL}(N, \mathbf{R})$ symmetry. This is motivated by the aim to eventually describe a quantum mechanical object with a fixed set of beables (related specifically to $\vec{A}$ ) in the deterministic model. Therefore, we would like the solution $F$ to be left invariant only by the subgroup of $\operatorname{GL}(N, \mathbf{R})$ which effects permutations of the $\hat{A}_{n}$ 's. Yet, 
it must remain invariant under unitary transformations in Hilbert space. This leads us to the initial condition:

$$
\begin{aligned}
F\left(M, t_{0}\right) & \equiv f\left(\vec{\omega}\left(t_{0}\right)-M \cdot \vec{A}\right) \\
& \equiv \sum_{n=1}^{N} \prod_{j=1}^{d}\left[\sum_{m=1}^{N} M_{n m} A_{m}^{j}-\omega_{n}\left(t_{0}\right)\right]^{2}=\sum_{n=1}^{N} \operatorname{det}^{2}\left[M \cdot \vec{A}-\vec{\omega}\left(t_{0}\right)\right]_{n},
\end{aligned}
$$

where the last sum is over the components of the vector inside [...] and the determinant refers to a $d$-dimensional Hilbert space on which the operators act that are collected in $\vec{A}$. - The evolving solution is then simply obtained by replacing $t_{0}$ by $t$ here, with $\vec{\omega}(t)$ still to be determined.

It is important to realize that operators and Hilbert space have only been introduced for convenient bookkeeping. Essentially needed, so far, are arbitrary real numbers $A_{n}^{j}$ which parametrize the initial condition, as in Eqs. (16)-(17).

Furthermore, we remark that the sum of squares of determinants in Eq. (17) is zero, if and only if the $N$-dimensional vector $\vec{\omega}$ corresponds to one of the points of the $N$-dimensional finite lattice defined by the $d \times N$ numbers $A_{n}^{j}$ or, rather, by the $d$ eigenvalues of each one of the $N$ operators $M \cdot \hat{A}$. In this way, the initial condition here presents a generalization of the function $f(\omega)$ of 't Hooft's existence theorem, cf. Eqs. (3) .

Finally, a boundary condition on the solution for the field $F$ is imposed:

$$
\frac{\mathrm{d} \vec{\omega}(t)}{\mathrm{d} t}=-\kappa \frac{\partial}{\partial \vec{\omega}} F^{2}\left(M^{*}, t\right)
$$

with $\kappa>0$, and where $M^{*} \in \mathrm{GL}(N, \mathbf{R})$ is fixed but arbitrary. This equation simultaneously determines $\vec{\omega}$, once its initial value $\vec{\omega}\left(t_{0}\right)$ is supplied.

For the solution specified by the above initial condition, Eqs. (16)-(17), this boundary condition suitably generalizes Eq. (3). Similarly to our discussion in the Introduction, it is easy to see that the zeros of $F$ (corresponding to points on the $d \times N$ lattice above) are attractive. Thus, the vector $\vec{\omega}$ is attracted to a fixed point:

$$
\omega_{n}(t) \stackrel{t \rightarrow \infty}{\longrightarrow} \sum_{m=1}^{N} M_{n m}^{*} A_{m}^{j(m)}=: \omega_{n}^{*},
$$

i.e., to a fixed vector with components built from linear combinations of eigenvalues of the operators $\hat{A}_{m}$; which particular eigenvalues contribute, indexed by $j(m), j=1, \ldots, d$, depends on the arbitrary initial condition for $\vec{\omega}$, analogous to the case reviewed in the Introduction.

Furthermore, the field $F$ decays to zero on the boundary $\left(M=M^{*}\right)$ and approaches a constant value, $F(M, t \rightarrow \infty)=f\left(\vec{\omega}^{*}-M \cdot \vec{A}\right)$, elsewhere. Through the zeros of $F$ and depending on $\omega\left(t_{0}\right)$, asymptotically $\vec{\omega} \approx \vec{\omega}^{*}$ also defines a vector of eigenvalues for the $N$ operators $M \cdot \vec{A}$, simply given by $M \cdot\left(M^{*}\right)^{-1} \vec{\omega}^{*}$, for any choice of $M \in \operatorname{GL}(N, \mathbf{R})$. 
This completes the set-up of the deterministic model. Before we turn to the emergent quantum mechanical behaviour in the next section, let us briefly discuss here the role of the auxiliary field $F$.

Applying Ockham's razor, we should omit wave equation (13), since only Eqs. (12), (16)(17), and (18) are needed for what follows. However, only a field equation of motion allows to separately interpret Eqs. (16)-(17) and (18) as a symmetry breaking initial condition and a boundary condition, respectively. The boundary condition introduces dissipation which leads to the attractive periodic orbits of $\vec{\varphi}$ that will be essential for the quantum mechanical features. This may open a way to explain the dissipation as a dynamical effect of neglected degrees of freedom or nonlinearities that must come into play when one tries to deal with physical forces. The latter are still missing to some extent. Despite that all spectral information seems to arise from the initial condition (in the form of numbers $A_{n}^{j}$ ), their allowed values should be dynamically constrained, as well as the special symmetry breaking 2

\section{Emergent quantum mechanics}

The considerations of Eqs. (8) -(10) are now easily generalized for the case at hand.

Similarly as before, we consider prequantum states $\psi$ which describe the trajectory of the deterministic system for an arbitrary but fixed initial condition:

$$
\begin{aligned}
\psi(\vec{\varphi}, \vec{\omega} ; t) & =\sum_{\vec{n}} e^{i \vec{n} \cdot \vec{\varphi}} \psi_{\vec{n}}(\vec{\omega} ; t) \\
& \stackrel{t \rightarrow \infty}{\longrightarrow} \sum_{\vec{n}} e^{i \vec{n} \cdot\left(\vec{\varphi}-\vec{\omega}^{*} t\right)} \psi_{\vec{n}}\left(\vec{\omega}^{*} ; 0\right)
\end{aligned}
$$

where $\vec{\omega}^{*}$ is the fixed point to which $\vec{\omega}(t)$ is attracted, depending on its initial value $\vec{\omega}\left(t_{0}\right)$, as discussed in the previous section, and periodicity in $\vec{\varphi}$ underlies the Fourier transformation.

A new feature arises here in that the states fall into superselection sectors that can be classified by the absolutely conserved vector $\vec{n}$. Furthermore, the states are specified by the asymptotic vector $\vec{\omega}^{*}$. All its components contribute to the phase, which describes the evolution of the state. That is, all $N$ Hermitian operators $\sum_{m} M_{n m} \hat{A}_{m}$ contribute, each with a set of $d$ eigenvalues $\sum_{m} M_{n m} A_{m}^{j}$. Following a single realization of the deterministic trajectory, the resulting particular eigenvalue can be given by $\sum_{m, k} M_{n m}\left(M^{*}\right)_{m k}^{-1} \omega_{k}^{*}$, as before.

\footnotetext{
${ }^{2}$ Unless we are prepared to accept an accidental yet decisive influence of the initial conditions on the model universe and its emergent (quantum mechanical) laws. The view that a random Hamiltonian might serve as a starting point to explain emergent physical laws has recently been expressed in Ref. [16] (see also further references therein), studying consequences of the clock ambiguity in time reparametrization invariant theories.
} 
Three qualitatively different situations may arise. - First, the model universe may find itself in a state where all components of $\vec{n}$ are equal, denoted by $\vec{n}^{\prime} \equiv\left(n^{\prime}, \ldots, n^{\prime}\right)^{t}$. In this case, the Hamiltonian, which generates the evolution, must be identified as:

$$
\hat{H}=\sum_{m, k=1}^{N} M_{m k}^{*} \hat{A}_{k},
$$

which picks an eigenvalue $E_{*}=\sum_{m, k} M_{m k}^{*} A_{k}^{j(k)}=\sum_{m} \omega_{m}^{*}$, following a particular deterministic trajectory. Here, emergent quantum states are related to the prequantum states by:

$$
e^{-i E_{*} t^{\prime}} \psi\left(E_{*}\right)=e^{-i n^{\prime} \sum_{m} \omega_{m}^{*} t} \psi_{\vec{n}^{\prime}}\left(\vec{\omega}^{*} ; 0\right)
$$

with $t^{\prime}:=n^{\prime} t$, cf. Eq. (10). One of the beables, corresponding to $\omega_{1}^{*}$, for example, could be eliminated in favour of the Hamiltonian and $E_{*}$, respectively, such that the above relation becomes $\psi\left(E_{*}\right) \propto \psi_{\vec{n}^{\prime}}\left(E_{*}, \omega_{2}^{*}, \ldots, \omega_{N}^{*} ; 0\right)$. Thus, we find degenerate energy eigenstates, which are further resolved by the eigenvalues of the $N-1$ remaining beables, i.e., by the values of $\omega_{2}^{*}, \ldots, \omega_{N}^{*}$.

Second, assuming that the natural scales of all $\omega_{n}^{*}$ are of the same order of magnitude, one of the components of the superselection vector $\vec{n}$, say $n_{1}$, may be very much larger than all others. In this case, it seems natural to consider the Hamiltonian:

$$
\hat{H}_{\sim}=\sum_{k=1}^{N} M_{1 k}^{*} \hat{A}_{k},
$$

with eigenvalue(s) $E_{*}=\omega_{1}^{*}$, which presents a valid approximation, as long as only sufficiently small eigenvalues $\omega_{m>1}^{*}$ have to be taken into account, $\left|n_{1} \omega_{1}^{*}\right|>>\sum_{m=2}^{N}\left|n_{m} \omega_{m}^{*}\right|$. In this case:

$$
e^{-i E_{*} t^{\prime}} \psi\left(E_{*}\right)=e^{-i n_{1} \omega_{1}^{*} t} \psi_{\vec{n}}\left(E_{*}, \omega_{2}^{*}, \ldots, \omega_{N}^{*} ; 0\right)
$$

with $t^{\prime}:=n_{1} t$. That is, the contribution to the phase which is most sensitive to the evolution dominates all others. This leads again to degenerate energy eigenstates, which are resolved by the remaining beables.

There will be only accidental degeneracies, if any, of the emergent energy eigenstates in the third case, when all beables possibly contribute to the Hamiltonian:

$$
\hat{H}_{\text {all }}=\vec{n} \cdot M^{*} \cdot \vec{A},
$$

with eigenvalues of the form $E_{*}=\vec{n} \cdot \vec{\omega}^{*}$. Here, we obtain:

$$
e^{-i E_{*} t} \psi\left(E_{*}\right)=e^{-i \vec{n} \cdot \vec{\omega}^{*} t} \psi_{\vec{n}}\left(\vec{\omega}^{*} ; 0\right)
$$

where an arbitrary $\omega_{i}^{*}$ could be replaced by $E_{*}-\sum_{m \neq i} n_{m} \omega_{m}^{*} / n_{i}$, provided $n_{i} \neq 0$. Thus, in this most general case, there still exist a unique Hamiltonian and a related energy variable, which govern the evolution of the emergent states. However, all beables contribute in a simple but nontrivial way to these quantities. 


\section{Conclusion}

We have shown that 't Hooft's theorem - stating the existence of a deterministic model that reproduces the dynamics of a given quantum system - can be extended to cover objects that are characterized by sets of beables. The symmetry of beables, which we introduced, has been useful in formulating a correspondingly extended deterministic prequantum model.

As we discussed, the deterministic model building appears at a sort of kinematical stage, so far, since the initial and boundary data that we invoked ask for an explanation. Possibly, the large symmetry of beables will be an ingredient for a theory that explains the necessary dissipation mechanism and constrains the initial data - and, thus, the relevant beables - in a dynamical way.

\section{References}

[1] G. 't Hooft, Emergent Quantum Mechanics and Emergent Symmetries, presented at PASCOS 13, Imperial College, London, July 6, 2007; arXiv:0707.4568.

[2] G.'t Hooft, A mathematical theory for deterministic quantum mechanics, J. Phys.: Conf. Ser. 67 (2007) 012015; arXiv:quant-ph/0604008.

[3] H.-T. Elze, Int. J. Theor. Phys. 46, No 8 (2007) 2063-2081; arXiv:hep-th/0510267.

[4] H.-T. Elze, Deterministic models of quantum fields, J. Phys.: Conf. Ser. 33 (2006) 399-404; arXiv:gr-qc/0512016.

[5] S. L. Adler, "Quantum Mechanics as an Emergent Phenomenon" (Cambridge Univ. Press, Cambridge, 2005).

[6] F. Markopoulou and L. Smolin, Phys. Rev. D70 (2004) 124029.

[7] M. Blasone, P. Jizba and H. Kleinert, Braz. J. Phys. 35 (2005) 497.

[8] M. Blasone, P. Jizba and G. Vitiello, Phys. Lett. A287 (2001) 205.

[9] X. F. Liu and C.P.Sun, J. Math. Phys. 42 (2001) 3665.

[10] G. 't Hooft, Int. J. Theor. Phys. 42 (2003) 355.

[11] G. 't Hooft, Class. Quant. Grav. 16 (1999) 3263.

[12] G. 't Hooft, J. Stat. Phys. 53 (1988) 323. 
[13] J.S. Bell, "Speakable and Unspeakable in Quantum Mechanics", 2nd edition (Cambridge Univ. Press, Cambridge, 2004).

[14] R.D. Sorkin, Causal Sets: Discrete Gravity (Notes for the Valdivia Summer School), proceedings of the Valdivia Summer School, A. Gomberoff and D. Marolf (ed.); arXiv:gr-qc/0309009.

[15] D. P. Rideout and R. D. Sorkin, Phys. Rev. D63 (2001) 104011.

[16] A. Albrecht and A.Iglesias, The clock ambiguity and the emergence of physical laws; arXiv:0708.2743. 